to changing temperatures when carbon dioxide concentrations in the models are first increased and then gradually reduced to preindustrial levels. In Antarctica, however, sea ice returns at first, but had not recovered by the end of the simulation, even after a further 150 years of preindustrial $\mathrm{CO}_{2}$ levels.

This lack of ice recovery is a result of strong heat uptake by the Southern Ocean, which continues to warm parts of the seas around Antarctica long after global warming has been reversed, according to the authors.

Geophys. Res. Lett. http://doi. org/xh3 (2014)

\section{ANIMAL BEHAVIOUR}

\section{Electric eel zaps} neurons of its prey

The electric eel stuns its fish prey by emitting electrical pulses that control parts of the nervous system of its victim.

Kenneth Catania at

Vanderbilt University

in Nashville, Tennessee, studied the behaviour and electrical discharges of an eel (Electrophorus electricus; pictured) when it was presented with fish in an aquarium. He found that the

eel's shocks immobilize the fish

by activating nerves controlling the muscles, causing them to contract throughout the fish's body even when the fish's brain and spinal cord were destroyed. When the fish was hidden, the eel sent out two quick pulses, causing the fish to twitch, followed soon by a high-voltage zap and an attack.

The results show how the electric eel can remotely control its prey.

Science 346, 1231-1234 (2014)

$$
\text { IMMUNOLOGY }
$$

\section{How immune cells search and destroy}

To locate the source of an infection, immune cells called neutrophils take directions from local blood cells.

Neutrophils are the first responders to an infection, where they produce pathogenkilling compounds. To determine how they home in on infections and other injuries, a team led by Andrés Hidalgo at Spain's National Centre for Cardiovascular Research in Madrid imaged blood vessels in live mice that were showing an inflammatory response.

The authors discovered that neutrophils drifting in the bloodstream stuck to blood vessel walls and then sent out arm-like extensions. When these encountered blood cells called platelets - which are activated by injury to help to stop bleeding - the neutrophils began to migrate along the vessel wall and churn out toxic chemicals. Blocking communication between neutrophils and platelets lessened tissue damage from excessive inflammation in mouse models of sepsis, lung injury or stroke.

Science 346, 1234-1238 (2014)

\section{NEUROSCIENCE}

\section{Injury blunts brain waste disposal}

Fluid channels in the brain that help to remove waste could be impaired after traumatic injury, promoting cell death.

After injury, brain cells release a protein called tau, which accumulates as tangles and is associated with neurodegeneration and dementia. Jeffrey Iliff at the Oregon Health and Science

\title{
SOCIAL SELECTION Romilumaticale
}

\section{Funders drawn to alternative metrics}

In the digital age, a growing number of researchers and publishers are using more than just citation counts to track the impact of their articles. In an essay in PLoS Biology, three authors from a major UK research-funding agency argue that alternative metrics - or altmetrics, such as social-media mentions - can help funders to measure the full reach of the research that they support. Some researchers have already used these metrics in their favour. On his lab blog, Fernando Maestre, an ecologist at King Juan Carlos University in Madrid, explained how he included altmetrics in a successful grant proposal earlier this year. But not everyone is convinced that the new metrics are good for science. John Gilleard, a veterinary parasitologist at the University of Calgary in Canada, asked on Twitter: "Will an increased emphasis on \#altmetrics pressure researchers to 'over hype' their results?" PLoS Biol. 12, e1002003 (2014)

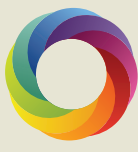

Based on data from altmetric.com. Altmetric is supported by Macmillan Science and Education, which owns Nature Publishing Group.

DNATURE.COM

For more on popular papers: go.nature.com/xep8gv

University, Portland, and

his colleagues

showed that tau

is cleared from young healthy mouse brains along the 'glymphatic pathway', channels that wash out waste from the brain.

The authors found that after traumatic injury, the pathway's performance decreased by about $60 \%$. It was reduced even further in injured mice in which a gene important for the pathway, aquaporin-4, had been knocked out. These mice developed tau tangles and performed less well in cognitive tests.

J. Neurosci. 34, 16180-16193 (2014)

\section{ANIMAL BEHAVIOUR}

\section{Some bats click wings to navigate}

Some bat species unable to use sonar to sense their environment can instead navigate using echoes from clicking their wings possibly an early, crude form of echolocation.

A team led by Arjan
Boonman and Yossi Yovel at Tel Aviv University in Israel studied three species of wild, non-echolocating Old World fruit bat (pictured is Cynopterus brachyotis). They found that individuals of two species emitted clicks more frequently in the dark than in the light, and could find and land on large objects, although they failed to detect small obstacles. When the researchers taped the bats' wings, the clicking stopped, but the exact clicking mechanism could not be determined.

The authors suggest that much can be learned about the evolution of echolocation from these fruit bats.

Curr. Biol. http://doi.org/xmr (2014)

\section{$\rightarrow$ NATURE.COM}

For the latest research published by Naturevisit:

www.nature.com/latestresearch 